\title{
General relativistic gravitational field of a rigidly rotating disk of dust: Solution in terms of ultraelliptic functions
}

\author{
G. Neugebauer and R. Meinel \\ Max-Planck-Gesellschaft, Arbeitsgruppe Gravitationstheorie \\ an der Universität Jena, Max-Wien-Platz 1, D-07743 Jena, Germany
}

\begin{abstract}
In a recent paper we presented analytic expressions for the axis potential, the disk metric, and the surface mass density of the global solution to Einstein's field equations describing a rigidly rotating disk of dust. Here we add the complete solution in terms of ultraelliptic functions and quadratures.
\end{abstract}

04.20.Jb, 04.40.-b, 95.30.Sf 
a. Introduction. An infinitesimally thin disk of dust rotating uniformly around its symmetry axis is the simplest model of a rotating self-gravitating body. Within Newton's theory of gravitation this model is described by the Maclaurin solution of the Laplace equation. In the present letter we show that the model of a uniformly rotating disk of dust allows for an explicit analytic solution in Einstein's theory of gravitation as well. This seems to be the first rigorous global solution of the rotating body problem (for perfect fluids with $p=0$ ) in general relativity.

An approximate solution of the problem was given by Bardeen and Wagoner [1], [2]. By applying the Inverse (Scattering) Method to the related boundary value problem of the Ernst equation we found the solution in terms of two linear integral equations, a 'small' and a 'big' one [3]. The analytic solution to the small integral equation in terms of the Weierstrass function led to explicit expressions for the Ernst potential on the symmetry axis, the disk metric, and the surface mass density [4]. In the following we will show that the complete solution of the problem may be represented, up to quadratures, in terms of ultraelliptic functions.

b. The metric. The line element reads in Weyl-Lewis-Papapetrou-coordinates:

$$
d s^{2}=e^{-2 U}\left[e^{2 k}\left(d \rho^{2}+d \zeta^{2}\right)+\rho^{2} d \varphi^{2}\right]-e^{2 U}(d t+a d \varphi)^{2} .
$$

(We use units where Newton's gravitational constant $G$ as well as the velocity of light $c$ are equal to 1.) The metric functions $e^{2 U}, e^{2 k}$, and $a / \rho_{0}$ depend uniquely on the normalized coordinates $\rho / \rho_{0}, \zeta / \rho_{0}$, and the parameter

$$
\mu=2 \Omega^{2} \rho_{0}^{2} e^{-2 V_{0}}
$$

where $\Omega, \rho_{0}$ and $V_{0}$ are the angular velocity, the coordinate radius, and the 'surface potential' $V_{o}(\mu) \equiv U(\rho=0, \zeta=0, \mu)$, respectively. The disk is characterized by $\zeta=0,0 \leq \rho \leq \rho_{0}$.

c. The Ernst potential. The (complex) Ernst potential is defined by

$$
f=e^{2 U}+\mathrm{i} b,
$$

where the imaginary part $b$ is related to the metric function $a$ according to

$$
a_{, \rho}=\rho e^{-4 U} b_{, \zeta} ; \quad a_{, \zeta}=-\rho e^{-4 U} b_{, \rho}
$$

i. e., a may be calculated from a given Ernst potential (and its derivatives) by a quadrature. The same holds for the metric function $e^{2 k}$. Hence, an axisymmetric stationary solution to the vacuum Einstein equations is sufficiently characterized by its Ernst potential. Note that our global solution is completely described by the vacuum solution outside the disk including the boundary data on the disk.

The Ernst potential for the dust disk solution is given by the expression

$$
f=\exp \left\{\mu\left[\int_{X_{1}}^{X_{a}} \frac{X^{2} d X}{W}+\int_{X_{2}}^{X_{b}} \frac{X^{2} d X}{W}-\int_{-i}^{\mathrm{i}} \frac{h X^{2} d X}{W_{1}}\right]\right\},
$$

with

$$
W=W_{1} W_{2}, \quad W_{1}=\sqrt{\left(X-\zeta / \rho_{0}\right)^{2}+\left(\rho / \rho_{0}\right)^{2}}, \quad W_{2}=\sqrt{1+\mu^{2}\left(1+X^{2}\right)^{2}}
$$




$$
\begin{gathered}
h=\frac{\ln \left(\sqrt{1+\mu^{2}\left(1+X^{2}\right)^{2}}+\mu\left(1+X^{2}\right)\right)}{\pi \mathrm{i} \sqrt{1+\mu^{2}\left(1+X^{2}\right)^{2}}}, \\
X_{1}^{2}=\frac{\mathrm{i}-\mu}{\mu}, \quad X_{2}^{2}=-\frac{\mathrm{i}+\mu}{\mu} \quad\left(\Re X_{1}<0, \quad \Re X_{2}>0\right) .
\end{gathered}
$$

The upper integration limits $X_{a}$ and $X_{b}$ in the first two integrals are hyperelliptic functions of two variables $u$ and $v$ (i. e. 'ultraelliptic' functions) defined by

$$
\int_{X_{1}}^{X_{a}} \frac{d X}{W}+\int_{X_{2}}^{X_{b}} \frac{d X}{W}=u, \quad \int_{X_{1}}^{X_{a}} \frac{X d X}{W}+\int_{X_{2}}^{X_{b}} \frac{X d X}{W}=v,
$$

where the variables $u$ and $v$ are given by

$$
u=\int_{-\mathrm{i}}^{\mathrm{i}} \frac{h d X}{W_{1}}, \quad v=\int_{-\mathrm{i}}^{\mathrm{i}} \frac{h X d X}{W_{1}} .
$$

The third integral in (5) as well as the integrals in (10) have to be taken along the imaginary axis in the complex $\mathrm{X}$-plane with $W_{1}$ and $h$ fixed according to $\Re W_{1}<0$ (for $\rho, \zeta$ outside the disk) and $\Re h=0$.

Eqs. (9) have exactly the form of Jacobi's famous inversion problem [5], which was solved by Göpel [6] and Rosenhain [7] who were able to express $X_{a}(u, v)$ and $X_{b}(u, v)$ in terms of (ultraelliptic) theta functions. In our case the moduli of the theta functions depend on $\rho / \rho_{0}$, $\zeta / \rho_{0}$, and $\mu$.

It should be noted that the integrations from $X_{1}$ to $X_{a}$ resp. $X_{2}$ to $X_{b}$ in (5) and (9) have to be performed along the same curve in the two-sheeted Riemann surface associated with $W(X)$. This leads to a unique Ernst potential $f\left(\rho / \rho_{0}, \zeta / \rho_{0}, \mu\right)$. More technical details and final expressions for the metric in terms of theta functions will be presented in a subsequent paper.

d. The Newtonian limit $\mu \ll 1$. At $\mu=0$, the solution may be expanded into a power series in $\mu^{1 / 2}$ :

$$
f=1+\sum_{n=1}^{\infty} f_{n} \mu^{(n+1) / 2} .
$$

The coefficients $f_{n}\left(\rho / \rho_{0}, \zeta / \rho_{0}\right)$ turn out to be elementary functions. A particularly simple result is obtained for $f_{1}$ to $f_{4}$. From Eqs. (5) to (10) we get

$$
X_{a}-X_{1}=\mathcal{O}\left(\mu^{3 / 2}\right), \quad X_{b}-X_{2}=\mathcal{O}\left(\mu^{3 / 2}\right),
$$

and

$$
f=\exp \left\{-\mu \int_{-\mathrm{i}}^{\mathrm{i}} \frac{h\left(X-X_{1}\right)\left(X-X_{2}\right) d X}{W_{1}}+\mathcal{O}\left(\mu^{3}\right)\right\} .
$$


Expanding $h, X_{1}$ and $X_{2}$ one can easily determine $f_{1}$ to $f_{4}$. (Note that $X=\mathcal{O}(1)$ in this integral.) The result for $f_{1}$ is

$$
f_{1}=\frac{1}{\pi \mathrm{i}} \int_{-\mathrm{i}}^{\mathrm{i}} \frac{\left(1+X^{2}\right) d X}{W_{1}}=-\frac{1}{\pi}\left\{\frac{4}{3} \cot ^{-1} \xi+\left[\xi-\left(\xi^{2}+\frac{1}{3}\right) \cot ^{-1} \xi\right]\left(1-3 \eta^{2}\right)\right\}
$$

with elliptic coordinates $\xi, \eta$ defined by

$$
\rho=\rho_{0} \sqrt{1+\xi^{2}} \sqrt{1-\eta^{2}}, \quad \zeta=\rho_{0} \xi \eta, \quad 0 \leq \xi<\infty, \quad-1 \leq \eta \leq 1 .
$$

Now, the Newtonian limit $U_{M l}$ can be read off from the first order relation

$$
g_{t t}=-\Re f=-\left(1+\mu f_{1}\right)=-\left(1+\frac{2 U_{M l}}{c^{2}}\right) .
$$

Obviously, $U_{M l}=c^{2} \mu f_{1} / 2$ is the Maclaurin solution, i. e. the Newtonian solution of the problem of a rigidly rotating disk of dust. Note that in this limit

$$
\mu=-\frac{2 U_{M l}(\rho=0, \zeta=0)}{c^{2}}=\frac{2 \Omega^{2} \rho_{0}^{2}}{c^{2}} .
$$

(For the sake of clarity we have reintroduced the velocity of light $c$ in the last two equations.)

e. Discussion. In the parameter range $0<\mu<\mu_{0}=4.62966184 \ldots$ [4] the solution is asymptotically flat and regular everywhere outside the disk. Its baryonic surface mass density is positive and vanishes at the rim of the disk. For $\mu \rightarrow \mu_{0}$ ( $\Omega$ finite) and $\rho^{2}+\zeta^{2} \neq 0$ it approaches exactly the extreme Kerr solution (mass $M=1 / 2 \Omega$ ). The meaning of the solution for $\mu>\mu_{0}$ deserves further discussion.

The analytic expressions above allow for a detailed investigation of characteristic properties, as for instance the formation of toroidal ergospheres, dragging effects, motion of test particles, and stability. 


\section{REFERENCES}

[1] J. M. Bardeen and R. V. Wagoner, Astrophys. J. 158, L65 (1969).

[2] J. M. Bardeen and R. V. Wagoner, Astrophys. J. 167, 359 (1971).

[3] G. Neugebauer and R. Meinel, Astrophys. J. 414, L97 (1993).

[4] G. Neugebauer and R. Meinel, Phys. Rev. Lett. 73, 2166 (1994)

[5] C. G. J. Jacobi, Crelle's Journ. f. Math. 9, 394 (1832).

[6] A. Göpel, Crelle's Journ. f. Math. 35, 277 (1847).

[7] G. Rosenhain, Crelle's Journ. f. Math. 40, 319 (1850). 